\title{
FIXAÇÃO DE CARBONO E A EMISSÃO DOS GASES DE EFEITO ESTUFA NA EXPLORAÇÃO DA CANA-DE-AÇÚCAR
}

\author{
Fixing of carbon and emission of greenhouse gases in the exploitation of sugar cane
}

\author{
Mauro de Paula ${ }^{1}$, Francisco Assis Rolim Pereira ${ }^{2}$, Edison Rubens Arrabal Arias ${ }^{2}$, \\ Bruno Ricardo Scheeren², Celso Correia de Souza², Danúbia Sales da Mata ${ }^{3}$
}

\begin{abstract}
RESUMO
A produção de uma tonelada $(\mathrm{t})$ de fitomassa em matéria seca (MS) de cana-de-açúcar fixa, no mínimo, 0,42 t em carbono (C), o que corresponde a mitigar 1,54 t de dióxido de carbono $\left(\mathrm{CO}_{2}\right)$ da atmosfera. Neste trabalho, objetivou-se efetuar um levantamento da quantidade de fitomassa da cana-de-açúcar produzida em 1 ha anualmente. Além de analisar o total de C fixado e a emissão de diversos gases de efeito estufa (GEE), em $\mathrm{CO}_{2}$ equivalente $\left(\mathrm{eqCO}_{2}\right)$, em consequência da adubação nitrogenada; da queima da fitomassa na colheita e da oxidação de combustíveis fósseis usados na produção, colheita e no transporte da cana até a indústria. Com base na análise dos dados, concluiu-se que ao adotar como procedimento a colheita da cana-de-açúcar crua, o produtor canavieiro estará deixando de emitir 0,286 tha- ano ${ }^{-1}$ de material particulado, 13,53 $\mathrm{t} \mathrm{ha}^{-1} \mathrm{ano}^{-1} \mathrm{em} \mathrm{eqCO}$ de outros gases, além de fixar o $\mathrm{C}$ na fitomassa, gerando um ativo ambiental de $52,50 \mathrm{t} \mathrm{ha}^{-1} \mathrm{ano}^{-1} \mathrm{de} \mathrm{eqCO}_{2}$. Ao somar-se o total da fixação, mais a redução que deixará de ser emitida, a mitigação total será de $66,03 \mathrm{t} \mathrm{ha}^{-1} \mathrm{ano}^{-1}$ de eqCO ${ }_{2}$.
\end{abstract}

Termo de indexação: Fitomassa, equivalência em dióxido de carbono, sustentabilidade.

\section{ABSTRACT}

The production of one tonne $(t)$ of phytomass in dry matter $(\mathrm{DM})$ of sugar cane assimilates at least $0.42 \mathrm{t}$ in carbon $(\mathrm{C})$ which corresponds to $1.54 \mathrm{t}$ of carbon dioxide $\left(\mathrm{CO}_{2}\right)$ from the atmosphere. This work aimed to make a survey of the quantity of phytomass

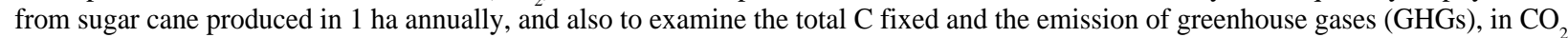
equivalent as a consequence of nitrogen fertilization, burning of phytomass at harvest and the oxidation of fossil fuels during production, harvest, and transport of the sugar cane to the industrial plant. Based on the analysis of data, it was concluded that by harvesting the sugar cane without burning, the farmer will not emit $0.286 \mathrm{t} \mathrm{ha}^{-1} \mathrm{year}^{-1}$ of particulate matter, $13,53 \mathrm{tha}^{-1} \mathrm{year}^{-1}$ in eqCO $_{2}$ of other gases. This will also assimilate carbon in the phytomass, generating an environmental active of $52,50 \mathrm{t} \mathrm{ha}^{-1} \mathrm{year}^{-1} \mathrm{of} \mathrm{eqCO}_{2}$. By adding up the total fixation and the reduction of emissions, the mitigation will total $66,03 \mathrm{tha}^{-1}$ year-1 of eqCO ${ }_{2}$.

Index terms: Phytomass, equivalence in carbon dioxide, sustainability.

(Recebido em 19 de setembro de 2008 e aprovado em 13 de maio de 2009)

\section{INTRODUÇÃO}

A atmosfera é composta por gases e vários deles interferem nas radiações tanto as emitidas pelo Sol, quanto as irradiadas pela Terra, Alguns, diretamente, por meio da absorção ou reflexão, como o dióxido de carbono $\left(\mathrm{CO}_{2}\right)$, o metano $\left(\mathrm{CH}_{4}\right)$, óxido nitroso $\left(\mathrm{N}_{2} \mathrm{O}\right)$, já, outros, indiretamente, como os hidrofluorcarbonos (HFCs) que destroem o ozônio permitindo a passagem de ondas solares como os raios ultravioleta. Essa absorção ou reflexão pelos gases é chamada de efeito estufa e atuam na manutenção do clima em nosso planeta, evitando temperaturas extremas (Yu, 2004).

O aumento da produção de alimentos, implica no aumento da mecanização e da queima de combustível. Existem incertezas nas estimativas dos fluxos de $\mathrm{CO}_{2}$ anual, porém a emissão dos combustíveis fósseis gira em torno de 5,5 a $6 \mathrm{PgC}\left(1 \mathrm{PgC}=10^{15} \mathrm{~g}=10\right.$ bilhões de toneladas $)$, e relacionado com as mudanças no uso da terra, são emitidos entre 1,6 e 2,3 PgC. Por outro lado, existem os sumidouros formados pelos oceanos, que absorvem entre 2,0 e 2,8 $\mathrm{PgC}$; pelas florestas, de 0,6 a 1,2 $\mathrm{PgC}$ e sumidouros residuais ou não determinados, de 1,3 a 2,4 PgC. Portanto, segundo os cálculos entre a entrada e a saída, restam entre 3,2 e 3,4 $\mathrm{PgC}$ anualmente, com tendência a aumentar (Aragão \& Shimabukuro, 2004).

Conforme o Protocolo de Kyoto, do qual o Brasil é signatário, os gases de efeito estufa (GEE), devem ter sua emissão reduzida em pelo menos $5 \%$ dos níveis emitidos em 1990, a ser atingido no primeiro período de compromisso (2008-2012). Visando a facilitar o objetivo do tratado, no artigo $3^{\circ}$, ficou estabelecido que a medição dos GEE seriam

\footnotetext{
1Universidade Anhanguera/UNAES - Campo Grande, MS - maurodepaula2008@gmail.com

2Universidade Anhanguera/UNIDERP - Campo Grande, MS

3Universidade Federal do Mato Grosso do Sul/UFMS - Campo Grande, MS
} 
calculados no potencial de equivalência ao dióxido de carbono $\left(\mathrm{eqCO}_{2}\right)$, ou seja, além do $\mathrm{CO}_{2}$ também o $\mathrm{CH}_{4}$, o $\mathrm{N}_{2} \mathrm{O}$, os HFCs, os PFCs e o $\mathrm{SF}_{6}$ (Cunha, 2005).

A Agenda 21, da qual o Brasil também é signatário, em seu capítulo 14, cujo subtítulo é "Promoção de Desenvolvimento Rural e Agrícola Sustentável”, salienta como principal objetivo o aumento da produção, sem descartar, a manutenção e o aperfeiçoamento da capacidade das terras agrícolas. Nas terras com menor potencial, buscase conservar ou ainda reabilitar, visando sempre a manter uma razão homem/terra sustentável (Brasil, 2005).

A cana-de-açúcar (Sacchharum officinarum L.) é uma planta perene da família Poaceae, ela fixa em torno de $100 \mathrm{mg}$ de $\mathrm{CO}_{2}$ por $\mathrm{dm}^{2}$ de área foliar por hora. Produz uma elevada quantidade de matéria seca (MS), em um período de 365 dias, obtendo-se uma taxa de crescimento médio de $18 \mathrm{~g} \mathrm{em} \mathrm{um} \mathrm{m}^{2}$ por dia, equivalente a produção de $65,70 \mathrm{t}$ ha $^{-1}$ de colmos, em que a média mundial era de $53 \mathrm{t} \mathrm{ha}^{-1}$ (Rodrigues, 1995).

Castro (2008), avaliou a produção de fitomassa pela cana-de-açúcar (colmos, ponteiros e folhas laterais), analisou três variedades, colhendo em três períodos diferentes, e constatando que a produção média de massa verde foi de $144,98 \mathrm{t} \mathrm{ha}^{-1}$, a qual continha uma média de $124,03 \mathrm{t} \mathrm{ha}^{-1}$ de colmos, restando 20,95 $\mathrm{t} \mathrm{ha}^{-1}$ de palha das folhas laterais dos colmos e dos ponteiros. Por meio de analises em laboratório, verificou-se também que essa fitomassa dos colmos, os ponteiros e as folhas laterais, transformaram-se numa média de 43,62 $\mathrm{t} \mathrm{ha}^{-1} \mathrm{de} \mathrm{MS}$.

Em um experimento realizado nos anos de 1998 a 2000 por Campos (2003), foram coletados dados sobre a produção e colheita da cana crua em Latossolo, foi colhida uma média de $95 \mathrm{t} \mathrm{ha}^{-1}$ de colmos $(87,24 \%$ das plantas $) \mathrm{e}$ depositados no solo $13,90 \mathrm{t} \mathrm{ha}^{-1}$ de palha $(12,76 \%$ das plantas), anualmente. Em Neossolo, a média de produção de colmos foi $85 \mathrm{t} \mathrm{ha}^{-1}(87,00 \%$ das plantas) e depositados no solo $12,80 \mathrm{t} \mathrm{ha}^{-1}$ de palha $(13,00 \%$ das plantas), anualmente. Com as folhas secas dos colmos e os ponteiros, restaram sobre o solo no período, uma média de $14 \mathrm{tha}^{-1} \mathrm{de}$ MS e um total de $6 \mathrm{t} \mathrm{ha}^{-1}$ de C $(42,86 \%$ de $\mathrm{C}$ na MS).

Para cada tonelada de $\mathrm{C}$ fixado na fitomassa, corresponde o equivalente a uma mitigação de 3,67 t de $\mathrm{CO}_{2}$ da atmosfera (Yu, 2004; Nishi et al., 2005).

Segundo Alvarez et al. (2000), ao analisar o desenvolvimento das raízes com colheita da cana crua e colheita da cana queimada, coletando amostras durante o ciclo cultural, para a cana crua, obteve-se, no primeiro ano, uma média de 0,277 g de MS de raízes por $\mathrm{m}^{3}$ de solo. Já, para a queimada, a média de $0,2713 \mathrm{~g}$ de MS de raízes por $\mathrm{m}^{3}$ de solo. No segundo ano, a cana crua obteve uma média de 0,1981 g de MS de raízes por $\mathrm{m}^{3}$, já, na cana queimada, como o solo fica mais exposto, perde-se a umidade mais rápido e as plantas necessitam de um sistema radicular mais vigoroso, foi obtido uma média de 0,2246 g de MS de raízes por $\mathrm{m}^{3}$ de solo.

Ao queimar a fitomassa, são produzidos vários gases que o homem, ao ser exposto a esses gases, se em pequenas doses, perde os reflexos nervosos, tem o raciocínio lógico e a destreza manual reduzidos, assim como o aumento de sonolências. Se aspirado em grandes doses, provoca a morte. Os compostos de nitrogênio e enxofre provocam bronquites crônicas e, em longo prazo, enfisema pulmonar. Com a prática da queimada do canavial na colheita, para cada tonelada de cana, são emitidos 0,0005 t de $\mathrm{N}_{2} \mathrm{O}, 0,004 \mathrm{t}$ de material particulado (MP), 0,006 $\mathrm{t}$ de HFCs e 0,028 t de CO (Arbex, 2001).

Segundo Macedo et al. (2004), ao adotar o processo de queima da cana-de-açúcar antes da colheita, para cada t de colmo restam $145 \mathrm{~kg}$ de MS, e destes $101 \mathrm{~kg}$ são de palha. Queimando os $101 \mathrm{~kg}$ de palha estarão sendo emitidos $0,286 \mathrm{~kg}$ de $\mathrm{CH}_{4}$. Já, em relação ao $\mathrm{N}_{2} \mathrm{O}$, para cada $101 \mathrm{~kg}$ de palha queimada, são emitidos $0,00825 \mathrm{~kg}$ de $\mathrm{N}_{2} \mathrm{O}$.

$\mathrm{Na}$ erosão existem as perdas físicas que são a texturização do solo agrícola (perda da argila do solo), perda da espessura dos horizontes e da taxa de infiltração da água. Já, a perda química como a capacidade de troca catiônica (CTC) e a saturação por bases, indicam a manutenção ou a degradação dos solos agrícolas. Caso a palha seja queimada deixando o solo exposto, anualmente serão perdidos $20,20 \mathrm{t} \mathrm{ha}^{-1}$ de solo. Com a palha sendo incorporada ao solo, a perda será de 13,80 t ha-1 de solo. E, por fim, se a palha ficar sobre o solo serão perdidos $6,50 \mathrm{t}$ ha $^{-1}$ de solo pela erosão (Donzelli, 2007).

Outro problema é a compactação do solo, em um experimento conduzido por Severiano (2007), foi monitorado na cultura da cana-de-açúcar mecanizada, em dois tipos de solo, com colheita em três períodos distintos, com índices de umidade diferenciadas, e concluiu que deve ser levado em conta que cada tipo de solo será impactado em maior ou menor percentual, conforme o índice de umidade no solo, e isso provoca uma redução no volume dos poros ocupados pela água e o ar, afetando o desenvolvimento das plantas.

Por outro lado Voll (2005), explica que ao colher a cana crua, as folhas secas e verdes dos colmos, além dos ponteiros, formam uma camada de palha com espessura aproximada entre 8 e $12 \mathrm{~cm}$ sobre o solo, pesando $15 \mathrm{t} \mathrm{ha}^{-1} \mathrm{de}$ MS, que reduz a compactação superficial e a erosão, aumenta o teor de matéria orgânica, dentre vários outros benefícios como a melhora da CTC, favorecendo o 
microclima próximo ao solo, evitando mudanças bruscas na temperatura e mantendo a umidade por um período bem maior do que se ele estivesse exposto.

Segundo Macedo et al. (2004), observaram que aplicando adubo nitrogenado $(\mathrm{N})$ no solo, ocorre uma perda entre $0,5 \%$ e $1,5 \%$, em forma de $\mathrm{N}_{2} \mathrm{O}$. Na região do CentroSul do Brasil, na cana planta a média utilizada, é de $28 \mathrm{~kg} \mathrm{ha}^{-1}$ $\mathrm{ano}^{-1}$ de N. Para a cana soca, é utilizado $87 \mathrm{~kg} \mathrm{ha}^{-1} \mathrm{ano}^{-1}$ de N. Como a maioria dos fertilizantes $\mathrm{N}$, a sua matéria prima é formulada a base de $\mathrm{NH}_{3}$, foi usado o índice de cálculo 1,5 $\%$. No trato cultural da cana-de-açúcar no Centro-Sul do Brasil, usa-se uma parte na cana planta $\left(28 \mathrm{~kg} \mathrm{ha}^{-1} \mathrm{ano}^{-1} \mathrm{de}\right.$ $\mathrm{N}$ ), e quatro partes com cana soqueira (348 $\mathrm{kg} \mathrm{ha}^{-1} \mathrm{ano}^{-1} \mathrm{de}$ $\mathrm{N})$. Assim, foram utilizadas anualmente uma média ponderada de $75,2 \mathrm{~kg} \mathrm{ha}^{-1} \mathrm{ano}^{-1}$ de N. Ao multiplicar por 1,5\%, chegouse a emissão de $1,13 \mathrm{~kg} \mathrm{ha}^{-1}$ ano $^{-1}$ de $\mathrm{N}_{2} \mathrm{O}$.

Face ao exposto, considerando que existem pouquíssimas publicações disponíveis sobre o assunto, conduziu-se este trabalho, com o objetivo de levantar, analisar e organizar informações, envolvendo todo o processo de produção da cultura da cana-de-açúcar. Desde o preparo do solo, a colheita e o transporte da cana-deaçúcar até a usina de beneficiamento além de confrontar as emissões e as fixações em dióxido carbônico equivalente e constatar o saldo ativo ou passivo ambiental.

\section{MATERIAL E MÉTODOS}

A partir de pesquisa bibliográfica, fez-se uma triangulação dos dados, já tratados, a fim de organizar um levantamento de toda a fitomassa produzida pela cultura da cana-de-açúcar em um hectare, tendo em vista, qual foi o impacto negativo gerado ao meio ambiente, para produzir essa fitomassa em seu ciclo cultural.

Para tanto, foram seguidas as seguintes fases: a) Pesquisa bibliográfica em trabalhos confiáveis e relevantes; b) Foi analisado, e selecionado o material disponível, nas seguintes categorias: passivos e ativos ambientais;

Os Passivos Ambientais: No relatório do IPCC (2007), demonstram-se os cálculos de conversão dos gases de efeito estufa (GEE), a fim de transformá-los em dióxido de carbônico equivalente $\left(\mathrm{eqCO}_{2}\right.$ ), é apresentado conforme a Tabela 1. Ela demonstra o Potencial de Aquecimento Global (PAG), onde o $\mathrm{CO}_{2}$ tem o parâmetro 1 ou referência (PAG 1), já o $\mathrm{CH}_{4}$ tem o PAG 21, portanto, para se produzir esse hectare de cana-de-açúcar o quanto de combustível fóssil foi consumido, e o quanto de GEE foram emitidos e convertidos em dióxido carbônico equivalente $\left(\mathrm{eqCO}_{2}\right)$, conforme Tabelas 2, 3, 6 e 7, além da quantidade de adubo nitrogenado consumido no ciclo cultural (Macedo et al., 2004). Ao adotar-se a prática de queimar a cultura antes da colheita, quais os GEE que foram emitidos e também convertidos em eqCO $\mathrm{CO}_{2}$, conforme seu PAG, demonstrado na Tabela 5 (Campos, 2003).

Os Ativos Ambientais: Na cultura da cana-de-açúcar o quanto foi produzido por hectare de fitomassa (colmos, ponteiros, folhas laterais e raízes). Focou-se a pesquisa na região Centro-Sul do Brasil, com os últimos dados de produção disponíveis (Carvalho, 2007), que foi de 366.320 .250 t na safra 2006/07. Buscou-se a área utilizada (INPE, 2007), que foi de 5.115 .590 ha, e foi obtida a média de produção da cana-de-açúcar na região do Centro-Sul por hectare na safra 2006/07, conforme Tabela 4. Como estes dados são somente dos colmos, também se buscou dados de produção das folhas laterais e dos ponteiros (Campos, 2003; Castro, 2008) e das raízes (Alvarez at al., 2000). Foi levantado o percentual de matéria seca (MS), nessa fitomassa (Castro, 2008). Na MS levantou-se o percentual de $\mathrm{C}$ fixado (Campos, 2003), conforme Tabela 8, e este resultado foi multiplicado por 3,67, que é o quanto de $\mathrm{CO}_{2}$ é retirado da atmosfera ao se fixar o $\mathrm{C}$ na fitomassa (Yu, 2004; Nishie et al., 2005).

Tabela 1 - IPCC - Global Warming Potential - (GWP-PAG).

\begin{tabular}{|c|c|c|c|}
\hline Nome do Gás & Fórmula Química & PAG. & Transf.em t de eqCO $\mathrm{CO}_{2}$ \\
\hline Gás metano & $1 \mathrm{t} \mathrm{de} \mathrm{CH}_{4}$ & PAG 21 & $21 \mathrm{t} \mathrm{de} \mathrm{eqCO}{ }_{2}$ \\
\hline Óxido nitroso & $1 \mathrm{tde} \mathrm{N}_{2} \mathrm{O}$ & PAG 310 & 310 t de eqCO ${ }_{2}$ \\
\hline Hidrofluorcarbono & $1 \mathrm{t}$ de $\mathrm{HFC}_{23}$ & PAG 11.700 & $11.700 \mathrm{t}$ de eqCO${ }_{2}$ \\
\hline Hidrofluorcarbono & $1 \mathrm{t}$ de $\mathrm{HFC}_{125}$ & PAG 2.800 & $2.800 \mathrm{t}$ de eqCO${ }_{2}$ \\
\hline Hidrofluorcarbono & $1 \mathrm{t}$ de $\mathrm{HFC}_{134 \mathrm{a}}$ & PAG 1.300 & $1.300 \mathrm{t}$ de eqCO $\mathrm{CO}_{2}$ \\
\hline Perfluorcarbono & $1 \mathrm{t} \mathrm{de} \mathrm{PFC}_{4}$ & PAG 6.500 & $6.500 \mathrm{t}$ de eqCO${ }_{2}$ \\
\hline Perfluorcarbono & $1 \mathrm{t}$ de $\mathrm{PF}_{2} \mathrm{C}_{6}$ & PAG 9.200 & $9.200 \mathrm{t}$ de eqCO${ }_{2}$ \\
\hline Perfluorcarbono & $1 \mathrm{t}$ de $\mathrm{PFC}_{318}$ & PAG 8.700 & $8.700 \mathrm{t}$ de eqCO${ }_{2}$ \\
\hline
\end{tabular}

Fonte: Adaptada de IPCC (2007). 
Por fim, confrontou-se o total dos Ativos e dos Passivos Ambientais gerados ao se produzir um hectare de cana-de-açúcar, ao erradicar a prática da queima da cultura ao colhê-la. Com isso, atendeu-se às expectativas iniciais, propostas pelo trabalho.

\section{RESULTADOS E DISCUSSÃO}

Como o plantio é realizado em, no máximo 1/5 (20\%) da área total, nela foram utilizados tratores agrícolas de porte médio, tanto na aplicação de calcário, quanto no controle químico. Foram utilizadas esteiras para as gradagens pesadas e subsolação; tratores agrícolas de porte grande para a sulcação e adubação do solo; caminhões para o transporte das mudas e distribuição da torta de filtro, que é aplicada em $30 \%$ da área total de plantio, conforme é visto na Tabela 2 :
Nos 4/5 restantes da área (80\%), são realizados apenas tratos culturais, utilizando tratores agrícolas de porte médio no aleiramento da palha, a aplicação de controle químico, cultivo mecânico e na irrigação da vinhaça, aplicando em $30 \%$ da área, conforme é visto na Tabela 3.

$\mathrm{Na}$ colheita mecânica, foram utilizadas máquinas que consomem em média 40,04 $\mathrm{L} \mathrm{h}^{-1}$, com uma produção de 45,00 $\mathrm{th}^{-1}$ de cana combinada (queimada e crua), então, o consumo é equivalente à colheita de 0,5461 ha ou 73,98 $\mathrm{L} \mathrm{ha}^{-1}$ de óleo (Macedo et al., 2004). Para o cálculo de eficiência dos veículos de três tipos (Truk 15 t, Romeu/Julieta $28 \mathrm{t} \mathrm{e} \mathrm{Treminhão} 45 \mathrm{t}$; consumindo, respectivamente, $30,30 \mathrm{ml} \mathrm{t}^{-1} \mathrm{~km}^{-1} ; 22,30 \mathrm{ml} \mathrm{t}^{-1}$ $\mathrm{km}^{-1} ; 18,50 \mathrm{ml} \mathrm{t}^{-1} \mathrm{~km}^{-1}$ ), a média ponderada dos três, levando em consideração distância da lavoura até a usina (cerca de $20 \mathrm{~km}$ ) e o consumo de cada tipo. No fim, obteve-se o resultado de 20,4 $\mathrm{ml} \mathrm{t}^{-1} \mathrm{~km}^{-1}$ de óleo diesel.

Tabela 2 - Consumo de Óleo Diesel para o Plantio da Cana-de-Açúcar.

\begin{tabular}{lccccc}
\hline Trato Cultural & Equipamento & Implemento & ha h$^{-1}$ & $\mathrm{~L} \mathrm{~h}^{-1}$ & $\mathrm{~L} \mathrm{ha}^{-1}$ \\
\hline Aplicaç.Calcário & Trator 78 CV & Carreta Distrib. & 1,61 & 6,00 & 3,73 \\
Errad. química & Trator 69 CV & Bomba Herbic. & 2,50 & 4,00 & 1,60 \\
Errad.Mecânica & Trator 143 CV & Eliminador. & 1,10 & 12,00 & 11,09 \\
Grade pesada I & Trator 165 CV & Grade 18dX34” & 1,30 & 27,60 & 21,23 \\
Subsolagem & Trator 165 CV & Subs. 5 Hastes & 1,00 & 26,00 & 26,00 \\
Grade pesada II & Trator 165 CV & Grade 18dX34” & 1,30 & 27,60 & 21,23 \\
Sulca e Adubar & Trator 170 CV & Sulc/Adub.Duplo & 1,10 & 15,00 & 13,64 \\
Transp. Mudas & Caminhão 360 CV & Trem/Rodotrem & 0,546 & 9,50 & 17,40 \\
Distrib. Mudas & Trator 69 CV & Carreta Distrib. & 0,60 & 4,00 & 6,67 \\
Apl.Ins./Cob.Suco & Trator 69 CV & Cobridor 2 linha. & 1,80 & 4,80 & 2,67 \\
Aplic. Herbicida & Trator 69 CV & Bomba Herbic. & 2,50 & 4,00 & 1,60 \\
Aplic. Torta Filt. & Caminhão 180 CV & 30\% da Área & 2,50 & 12,80 & 9,60 \\
Cultivo Mecânico & Trator 69 CV & Cultivador & 1,30 & 8,00 & 6,15 \\
\hline \multicolumn{7}{c}{ Para Plantar 1 ha de Cana-de-Açúcar o Consumo de Óleo Diesel é de: } \\
\hline \multicolumn{7}{c}{ A Reforma é Feita em 20 \% da Área Total (1/5), a Média Ponderada é de: } & $163,83 \mathrm{~L} \mathrm{ha}^{-1}$ \\
\hline
\end{tabular}

Fonte:- Adaptada de Macedo et al. (2004).

Tabela 3 - Consumo de Óleo Diesel na Manutenção Agrícola da Cana Soca.

\begin{tabular}{lccccc}
\hline Trato Cultural & Equipamento & Implemento & ha h & $\mathrm{L} \mathrm{h}^{-1}$ & $\mathrm{~L} \mathrm{ha}^{-1}$ \\
\hline Aleira/to da Palha & Trator 69 CV & Aleira/ de Palha & 1,50 & 4,00 & 2,67 \\
Cultivo Mecânico & Trator 143 CV & Cultivador. & 1,30 & 9,20 & 7,08 \\
Vinhaça(Trans+Apl) & Caminhão 180 CV & 30\% da Área & 2,50 & 61,74 & 7,41 \\
Aplicaç. Herbicida & Trator 69 CV & Bomba Herbic. & 2,50 & 4,00 & 1,60 \\
\hline \multicolumn{2}{l}{ Para manter 1 ha de cana-de-açúcar o consumo de óleo diesel é de: } & & & $18,75 \mathrm{~L} \mathrm{ha}^{-1}$ \\
\hline \multicolumn{2}{l}{ A manutenção é feita em 80 \% da área total (4/5), a média ponderada é de: } & & $15,00 \mathrm{~L} \mathrm{ha}^{-1}$ \\
\hline
\end{tabular}

Fonte: Adaptada de Macedo et al. (2004). 
Conforme é demonstrado na Tabela 4, a cana-deaçúcar na safra 2006/07, o Brasil atingiu uma produção total de 426.002.444 t (100\%), sendo que, destas, 53.250.700 $\mathrm{t}(12,50 \%)$ foram produzidas na região Norte-Nordeste e 372.751 .744 t (87,50 \%), na região Centro-Sul (Cavalho, 2007). Na região Centro-Sul, o estado do Espírito Santo atingiu uma produção total de 2.894.451 t (0,68 \%); o estado de Goiás, a produção total de 16.140.043 t (3,79\%); Mato Grosso, a produção total de 13.179 .510 t (3,09 \%); Mato Grosso do Sul, o total de 11.635 .096 t (2,73 \%); Minas Gerais, uma produção total de 29.034.195 t (6,82 \%); Paraná, um total de 31.994.581 t (7,51\%); Rio Grande do Sul, uma produção total de $91.919 \mathrm{t}(0,02 \%)$; Rio de Janeiro, um total de 3.445 .154 t $(0,81 \%)$; Santa Catarina que não produziu nada e, finalmente, São Paulo que produziu 264.336.825 t (62,05\%).

Ainda da Tabela 4, na safra 2006/07, para a região Centro-Sul ter atingido essa produção, foram necessários em torno de 6 milhões de ha. Apesar de não terem sido obtidos os dados dos seguintes Estados: Espírito Santo, Rio de Janeiro, Rio Grande do Sul e Santa Catarina, os demais utilizaram 5.115.590 ha, sendo que, destes, foram 4.729.483 ha para a safra normal e 386.107 ha necessitaram serem reformadas (INPE, 2007).

Como os GEE sobre a média de produção no CentroSul, na safra 2006/07, foi de 71,61 t ha-1, com isso, se queimada antes de colher, o material particulado atingiu 0,286 $\mathrm{tha}^{-1}$ ou 286,44 $\mathrm{kg} \mathrm{ha}^{-1}$ de M.P; o $\mathrm{N}_{2} \mathrm{O}$ ficou com
0,0358 $\mathrm{tha}^{-1}$ ou 35,80 $\mathrm{kg} \mathrm{ha}^{-1}$, o monóxido de carbono com $2 \mathrm{t} \mathrm{ha}^{-1}$ ou $2.005,08 \mathrm{~kg} \mathrm{ha}^{-1}$ de $\mathrm{CO}$ e o $\mathrm{CH}_{4}$ totalizou $20,48 \mathrm{~kg}$ $\mathrm{ha}^{-1}$. Para converter esse números em eqCO ${ }_{2}$, sobre os gases, foram aplicados os valores de conversão PAG, conforme Tabela 5.

Não foram obtidas informações precisas de quais os HPCs emitidos, ficaram fora dos cálculos, mas, deixaram de ser emitidos 429,66 $\mathrm{kg} \mathrm{ha}^{-1}$ de hidrocarbonetos, 286,44 $\mathrm{kg} \mathrm{ha}^{-1}$ de MP, além das 13,53 t ha-1 de eqCO $\mathrm{CO}_{2}$ contabilizados. Ainda reduziu a erosão, favoreceu o micro clima próximo ao solo evitando mudanças bruscas na temperatura, reteve a umidade por um período maior, além de manter ou até melhorar a CTC, das áreas agrícolas.

Para obter o consumo de óleo diesel; as práticas culturais e o consumo de óleo combustível necessários no plantio da cana-de-açúcar (em que o consumo ficou com a mesma média de 163,83 $\mathrm{L} \mathrm{ha}^{-1}$ de óleo diesel para as duas Tabelas, porém o plantio em apenas $1 / 5$ ou " $20 \%$ " da área, conforme a Tabela 2, e em 4/5 ou "80 \%" restantes de acordo com a Tabela 3), foram necessários realizar os tratos culturais com tratores agrícolas de porte médio, como o aleiramento da palhada, a aplicação de controle químico e o cultivo mecânico. Na irrigação com a vinhaça, aplicandoa em $30 \%$ da área, são consumidos em média $18,75 \mathrm{~L} \mathrm{ha}^{-1}$ de óleo diesel. Portanto, ao juntar o consumo do combustível com a cana planta $(20 \%)$ mais o consumo da cana soca $(80 \%)$, foi obtida uma média ponderada de 47,77 $\mathrm{L} \mathrm{ha}^{-1}$ de óleo diesel.

Tabela 4 - Área de Cana Safra e Reforma, Centro-Sul do Brasil - Safra 2006/07.

\begin{tabular}{cccccc}
\hline Estados & Safra (ha) & Reforma (ha) & Total (ha) & Produção (t) & Média t ha $^{-1}$ \\
\hline MS & 167.964 & 14.096 & 182.060 & 11.635 .096 & 63,91 \\
MT & 194.430 & 19.914 & 214.344 & 13.179 .510 & 61,49 \\
GO & 239.083 & 11.584 & 250.666 & 16.140 .043 & 64,39 \\
MG & 351.284 & 17.214 & 368.497 & 29.034 .195 & 78,79 \\
PR & 422.232 & 16.626 & 438.858 & 31.994 .581 & 72,90 \\
SP & 3.354 .490 & 306.674 & 3.661 .164 & 264.336 .825 & 72,20 \\
\hline Total & 4.729 .483 & 386.107 & 5.115 .590 & 366.320 .250 & 71,61 \\
\hline
\end{tabular}

Tabela 5 - Emissão de Gases de Efeito Estufa pela Queima da Palha.

\begin{tabular}{lrrr}
\hline Gás Efeito Estufa & Emissão em $(\mathrm{kg})$ & \multicolumn{1}{c}{ GWP ou PAG } & \multicolumn{1}{c}{$\mathrm{CO}_{2}$ Equivalente } \\
\hline $\mathrm{CO}$ & $2.005,08$ X & 1 & 2.005 \\
$\mathrm{CH}_{4}$ & $20,48 \mathrm{X}$ & $21=$ & 430 \\
$\mathrm{~N}_{2} \mathrm{O}$ & $35,80 \mathrm{X}$ & $310=$ & 11.098 \\
\hline TOTAL & & & $13,53 \mathrm{t} \mathrm{ha}^{-1} \mathrm{eqCO}_{2}$ \\
\hline
\end{tabular}


Por outro lado, ao fazer a colheita $100 \%$ mecânica, com máquinas que consomem em média 40,40 $\mathrm{L} \mathrm{ha}^{-1}$ ou 0,6733 L minuto, reduzindo a produtividade da máquina para $40,00 \mathrm{t} \mathrm{h}^{-1}$, pois o cálculo é para colher somente cana crua. Colhendo a média de produção obtida no Centro-Sul na

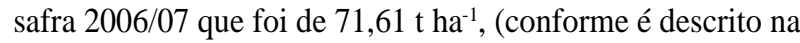
Tabela 6), em uma hora, colhe 0,5586 de 1 ha ou 1 ha colhe em 108 minutos. Multiplicado por 0,6733 L min., é obtido $72,72 \mathrm{~L} \mathrm{ha}^{-1}$ de óleo diesel. Com relação ao transbordo da cana para os caminhões, esses tratores gastam em média 9,0 $\mathrm{L} \mathrm{ha}^{-1}$ de óleo diesel e sua capacidade operacional é de 35,00 $\mathrm{t} \mathrm{ha}^{-1}$. Com a média de 71,61 t ha-1 em 1 hora transporta 0,4888 de 1 ha e consome 18,45 $\mathrm{L} \mathrm{ha}^{-1}$ de óleo diesel.

Como já visto, para transportar foi obtido o resultado de 20,4 $\mathrm{ml} \mathrm{t}^{-1} \mathrm{~km}^{-1}$ de óleo diesel, calculando com a produção obtida no Centro-Sul, na safra 2006/07, que foi 71,61 t ha-1 com uma distância média de 20 km, são consumidos 29,62 L de óleo diesel por ha. Juntando a cana, planta que ficou com uma média ponderada de $32,77 \mathrm{~L}$, com a cana soca que ficou com uma média ponderada de 15,77 L, a colheita mecânica da cana crua e transbordo, o consumo foi $91,17 \mathrm{~L}$, em 1 ha com 71,61 t de cana o consumo total de óleo diesel foi de 169,33 L.

Para calcular a emissão de eqCO do óleo diesel, sendo que cada L pesa $0,852 \mathrm{~kg}$; então $852 \mathrm{~g}$ multiplicado por $60 \mathrm{~g}$, o resultado dividido por $1000 \mathrm{ml}$, foi obtida a emissão de $5,112 \%$ de eqCO ${ }_{2}$, para cada $\mathrm{L}$ de óleo diesel na prospecção. Para cada $\mathrm{L}$ de óleo diesel são emitidos $5,112 \%$ em eqCO na prospecção, $22,15 \%$ de eqCO no refino, $1,7 \%$ de eqCO $\mathrm{CO}_{2}$ no transporte até o consumidor, $29,82 \%$ em eqCO $\mathrm{CO}_{2}$ pela evaporação e $268,38 \%$ em eqCO pela emissão direta (Macedo et al., 2004). Na Tabela 7, é convertido os 169,33 L de óleo diesel em eqCO, portanto, para plantar, cultivar, colher, realizar o transbordo e o transporte da produção da cana-de-açúcar até a indústria, com a queima de combustíveis fósseis, tanto pela emissão direta como indireta, será gerado um passivo de $554,00 \mathrm{~kg}$ $\mathrm{ha}^{-1}$ ano $^{-1}$ de eqCO . $_{2}$

Com o propósito de quantificar a emissão desse gás na produção da cana-de-açúcar com a adubação nitrogenada, são obtidas, anualmente, média ponderada de 75,2 $\mathrm{kg} \mathrm{ha}^{-1}$ ano $^{-1} \mathrm{de} \mathrm{N}$. Com uma evaporação de 1,5\%, chegou-se à emissão de $1,13 \mathrm{~kg} \mathrm{ha}^{-1} \mathrm{ano}^{-1}$ de $\mathrm{N}_{2} \mathrm{O}$. Se em eqCO $\mathrm{C}_{2}$, o $\mathrm{N}_{2} \mathrm{O}$ tem um PAG de 310 vezes, logo, foram emitidos $350,30 \mathrm{~kg} \mathrm{ha}^{-1}$ ano $^{-1}$ de eqCO . $_{2}$.

$\mathrm{Na}$ Tabela 8, são demonstrados os resultados obtidos, conforme Castro (2008), com os colmos "30\% de $71,61 \mathrm{t} \mathrm{ha}^{-1}$ corresponde a 21,48 $\mathrm{t} \mathrm{ha}^{-1}$ de MS"; com a palha, conforme Campos (2003), onde "71,61 tha ${ }^{-1}$ equivale 87,24 \%,

Tabela 6 - Consumo de Óleo Diesel com a Colheita Mecânica na Cana Crua.

\begin{tabular}{lccccc}
\hline Trato Cultural & Equipamento & Produção t h & ha h$^{-1}$ & $\mathrm{~L} \mathrm{~h}^{-1}$ & $\mathrm{~L} \mathrm{ha}^{-1}$ \\
\hline Colheita Mecân & Colheit 330 CV & 40,00 & 0,5586 & 40,40 & 72,72 \\
Transbordo & Trator 180 CV & 35,00 & 0,4888 & 9,00 & 18,45 \\
\hline \multicolumn{2}{c}{ Colher e trasbordo de 1 ha de cana-de-açúcar o consumo de óleo diesel é de: } & $91,17 \mathrm{~L} \mathrm{ha}^{-1}$ \\
\hline
\end{tabular}

Tabela 7 - Consumo de 169,33 L ha-1 de Diesel e a Emissão de $\mathrm{CO}_{2}$ Equivalente.

\begin{tabular}{|c|c|c|c|c|c|}
\hline Prospecção & Refino & Transporte & Evaporação & Emissão Direta & Emissão Total \\
\hline $\begin{array}{c}5,112 \% \mathrm{~L}^{-1} \\
\text { eqCO }_{2}\end{array}$ & $\begin{array}{c}22,15 \% \mathrm{~L}^{-1} \\
\mathrm{eqCO}_{2}\end{array}$ & $\begin{array}{c}1,70 \% \mathrm{~L}^{-1} \\
\mathrm{eqCO}_{2}\end{array}$ & $\begin{array}{c}29,82 \% \mathrm{~L}^{-1} \\
\text { eqCO }_{2}\end{array}$ & $\begin{array}{c}268,38 \% \mathrm{~L}^{-1} \\
\mathrm{eqCO}_{2}\end{array}$ & $327,17 \% \mathrm{~L}^{-1}$ de eqCO $\mathrm{CO}_{2}$ \\
\hline $8,66 \mathrm{~kg} \mathrm{eqCO} \mathrm{CO}_{2}$ & $37,51 \mathrm{~kg} \mathrm{eqCO}$ & $2,88 \mathrm{~kg} \mathrm{eqCO}{ }_{2}$ & $50,49 \mathrm{~kg} \mathrm{eqCO}{ }_{2}$ & $454,45 \mathrm{~kg}$ eqCO $\mathrm{CO}_{2}$ & $553,99 \mathrm{~kg} \mathrm{eqCO}{ }_{2}$ \\
\hline \multicolumn{5}{|c|}{ Em $169,33 \mathrm{~L}$ de óleo diesel, emitirá em eqCO $\mathrm{CO}_{2}$ um total de: } & $554 \mathrm{~kg}$ de eqCO${ }_{2}$ \\
\hline
\end{tabular}

Tabela 8 - Total de Matéria Seca e de Carbono na Fitomassa da Cana.

\begin{tabular}{|c|c|c|c|}
\hline Cana-de-açúcar & Fitomassa $\mathrm{t} \mathrm{ha}^{-1}$ de MS & $<\%$ de $\mathrm{C}$ na $\mathrm{MS}$ & $\mathrm{t} \mathrm{ha}^{-1}$ de C \\
\hline Colmos & $21,48 \mathrm{tha}^{-1}$ de MS X & $42,86 \%=$ & $9,21 \mathrm{t} \mathrm{ha}^{-1} \mathrm{de} \mathrm{C}$ \\
\hline Palhada & $10,47 \mathrm{tha}^{-1}$ de MS $\mathrm{X}$ & $42,86 \%=$ & $4,49 \mathrm{t} \mathrm{ha}^{-1} \mathrm{de} \mathrm{C}$ \\
\hline Raízes & $1,98 \mathrm{tha}^{-1}$ de MS $\mathrm{X}$ & $42,86 \%=$ & $0,85 \mathrm{t} \mathrm{ha}^{-1} \mathrm{de} \mathrm{C}$ \\
\hline TOTAL & $33,88 \mathrm{tha}^{-1}$ de MS $\mathrm{X}$ & $42,86 \%=$ & $14,55 \mathrm{t} \mathrm{ha}^{-1} \mathrm{de} \mathrm{C}$ \\
\hline
\end{tabular}

Ciênc. agrotec., Lavras, v. 34, n. 3, p. 633-640, maio/jun., 2010 
então os 12,76 \% restantes, equivale 10,47 t ha-1 de MS" e com as raízes, conforme Alvarez et al. (2000), foi de 1,98 t $\mathrm{ha}^{-1}$ de MS. Como o menor índice de $\mathrm{C}$ encontrado pelos pesquisadores na MS, foi de 42,86\%, conforme Campos (2003), tem-se um total em t ha-1 ano $^{-1}$ de C fixada pela fitomassa da cana-de-açúcar.

Ao multiplicar as $14,55 \mathrm{t} \mathrm{ha}^{-1}$ de $\mathrm{C}$, fixado pela fitomassa da cana crua, por 3,67 te $\mathrm{CO}_{2}$ retirado da atmosfera, o produtor canavieiro ao concluir o ciclo cultural da cana-de-açúcar, terá gerado um ativo ambiental com um crédito de 53,40 tha- ano $^{-1}$ de eqCO . $_{2}$.

De acordo com a cultura canavieira na safra 2006/ 07, na região do Centro-Sul, foi obtido uma média de fixação

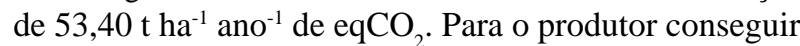
esse ativo ambiental, foi necessário impactar o meio ambiente com a adubação nitrogenada que emitiu $0,3503 \mathrm{t}$ ha $^{-1}$ ano $^{-1}$ de eqCO ${ }_{2}$, com a queima de combustíveis fósseis, emitindo $0,554 \mathrm{t} \mathrm{ha}^{-1}$ ano $^{-1}$ de eqCO ${ }_{2}$, num total passivo de $0,9043 \mathrm{t} \mathrm{ha}^{-1} \mathrm{ano}^{-1} \mathrm{de}$ eqCO . Ao confrontar o ativo de 53,40

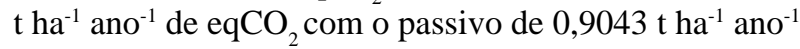
de eqCO $\mathrm{CO}_{2}$, o produtor ao concluir o ciclo cultural da canade-açúcar terá obtido um ativo ambiental de 52,50 t ha-1 $\mathrm{ano}^{-1}$ de eqCO

\section{CONCLUSÃO}

Com base na análise dos dados, concluiu-se que, ao adotar-se como procedimento, colher a cana-de-açúcar crua, o produtor canavieiro que erradicar a queima na colheita, deixará de emitir 0,286 tha- ano $^{-1}$ de $\mathrm{MP}, 13,53 \mathrm{t} \mathrm{ha}^{-1} \mathrm{ano}^{-1} \mathrm{em}$ eqCO $\mathrm{CO}_{2}$ de outros gases, além de fixar o $\mathrm{C}$ na fitomassa, gerando um ativo ambiental de 52,50 $\mathrm{t} \mathrm{ha}^{-1} \mathrm{ano}^{-1} \mathrm{de}$ eqCO, Ao somar-se o total da fixação $\left(52,50 \mathrm{t} \mathrm{ha}^{-1} \mathrm{ano}^{-1} \mathrm{de} \mathrm{eqCO}_{2}\right)$, mais a redução da emissão $\left(13,53 \mathrm{t} \mathrm{ha}^{-1}\right.$ ano $\left.^{-1} \mathrm{de}_{\mathrm{eqCO}}\right)$, a mitigação total será de $66,03 \mathrm{t} \mathrm{ha}^{-1}$ ano $^{-1}$ de eqCO ${ }_{2}$.

\section{REFERÊNCIAS BIBLIOGRÁFICAS}

ALVAREZ, I.A.; CASTRO, P.R.C.; NOGUEIRA, M.C.S. Crescimento de raízes de cana crua e queimada em dois ciclos. Scientia Agrícola, Piracicaba, v.57, n.4, p.653-659, out./dez. 2000.

ARAGÃO, L.E.O. e C.; SHIMABUKURO, Y.E. Perspectivas para o estudo da biogeoquímica do carbono em macro-escala integrando diferentes técnicas: modelagem ecológica, sensoriamento remoto e SIG. São José dos Campos: INPE, 2004. 97f. Relatório técnico do INPE-10731-RPQ/757.

ARBEX, M.A. Avaliação dos efeitos do material particulado proveniente da queimada da plantação da cana-de-açúcar sobre a morbidade respiratória na população de Araraquara-SP. 2001. 204f. Tese (Doutorado)-Faculdade de Medicina, Universidade de São Paulo, São Paulo, 2001.

BRASIL. Ministério do Meio Ambiente. A agenda 21. Disponível em: <http://www.mma.gov.bri index.php?ido=conteudomonta\&idEstrutura=18\&idConteudo=575;. Acesso em: 25 ago. 2007.

CAMPOS, D.C. Potencialidade do sistema de colheita sem queima da cana-de-açúcar para seqüestro de carbono. 2003. 103f. Tese (Doutorado)Escola Superior de Agricultura Luiz de Queiroz, Piracicaba, 2003.

CASTRO, H. S. de; ANDRADE, L. A. de B.; BOTREL, E. P.; EVANGELISTA, A. R. Rendimentos agricolas e forrageiros de três cultivares de cana-de-açucar (Saccharum spp.) em diferentes épocas de corte. Ciência e Agrotecnologia, v. 33, n. 5, p. 1336-1341, set./ out., 2009.

CARVALHO, E.P. Uma nova fonte de energia limpa para o mundo. 2007. Disponível em: <http://

iportalunica.com.br/portalunica/files/

portalunica.com.br/portalunica/fies/ referencia_publicacoes_informacoesunica-32. Arquivo.pdf . Acesso em: 14 abr. 2008.

CUNHA, K.B. Mecanismo de desenvolvimento limpo: evolução do instrumento e suas perspectivas. 2005. 198f. Dissertação (Mestrado)-Universidade Estadual de Campinas, Campinas, 2005.

DONZELLI, J.L. Erosão na cultura da cana-de-açúcar: situação e perspectivas. In: MACEDO, I.C. A energia da cana-de-açúcar: doze estudos sobre a agroindústria da cana-de-açúcar no Brasil e a sua sustentabilidade: 2.ed. São Paulo: Berlendis, 2007. 235p.

INPE. Tabela com os números da área de cana safra e reforma na região Centro-Sul - Safra 2006/2007. Disponível em: <http://Www.dsr.inpe.br/mapdsry tabelas.html'> Acesso em: 20 jun. 2007.

IPCC. Climate change 2007: technical summary: a report accepted by working group $i$ of the intergovernmental: panel on climate change. Disponível em: <http:// 'WwW.ipcc.ch/1>. Acesso em: 5 jul. 2008. 
MACEDO, I.C.; LEAL, M.R.L.V.; SILVA, J.E.A.R. Balanço das emissões de gases do efeito estufa na produção e no uso do etanol no Brasil. São Paulo: Secretaria do Meio Ambiente, 2004. 37p.

NISHI, M.H.; JACOVINE, L.A.G.; SILVA, M.L.; VALVERDE, S.R.; NOGUEIRA, H.P.; ALVARENGA. A.P. Influência dos créditos de carbono na viabilidade financeira de três projetos florestais. Revista Árvore, Viçosa, v.29, n.2, p.263-270, 2005.

RODRIGUES, J.D. Fisiologia da cana-de-açúcar. Botucatu: Unesp, 1995. 99p.
SEVERIANO, E.C.Indicadores de qualidade estrutural na avaliação da compactação do solo em decorrência da colheita mecanizada da cana-de-açúcar. 2007. 71f. Dissertação (Mestrado)-Universidade Federal de Lavras, Lavras, 2007.

VOLL, C.E. Aplicação de vinhaça e do extrato de palhiço de cana-de-açúcar no controle de plantas daninhas. 2005. 45f. Dissertação (Mestrado)-Escola Superior de Agricultura Luiz de Queiroz, Piracicaba, 2005.

YU, C.M. Seqüestro florestal de carbono no Brasil: dimensões políticas, socioeconômicas e ecológicas. São Paulo: Annablume IEB, 2004. 280p. 